\title{
Design and Analysis on Electric Performance of Threaded adhesive joints of composite Axial Insulation Break
}

\author{
Changchun Yang
}

College of Electric Engineering, Jiujiang University, China 332005

395254993@qq.com

Keywords: Threaded Adhesive joint, Insulation Break, Process, Electric Performance

Abstract. Threaded adhesive joints between stainless steel electrodes and insulating structure of axial insulation break was studied in detail. Wet winding process was chosen for Glass Fiber Reinforced Plastic inner insulation tube, and threaded adhesive method was chosen for joints bonding GRP to Stainless Steel. DC High voltage analysis showed the field strength of the axial break along the interface inner pipe is $1.23 \mathrm{kV} / \mathrm{mm}$, and the maximal field strength of the IBs locates on the top end of SS316L electrodes, the value of the maximum field strength is equal to $2.8 \mathrm{kV} / \mathrm{mm}$.

\section{Introduction}

Low-temperature high-voltage Helium tightness composite axial insulation breaks are key components of superconducting magnet systems [1]. Axial electrical insulation breaks are required to provide isolation between the cryogenic pipe-line potential and the coil potential. According to experience, if the mechanical properties are complete, then the electrical properties will be maintained[2].

This length ensures a dielectric strength along the inner pipe interface between high voltage and low voltage parts. DC High voltage test for the insulation breaks prototype, test design should be provided to meet the specified design voltage $29 \mathrm{kV}, 35 \mathrm{kV}$ and $56 \mathrm{kV}$, the design of these tests can also determine the scope of the breakdown field strength.

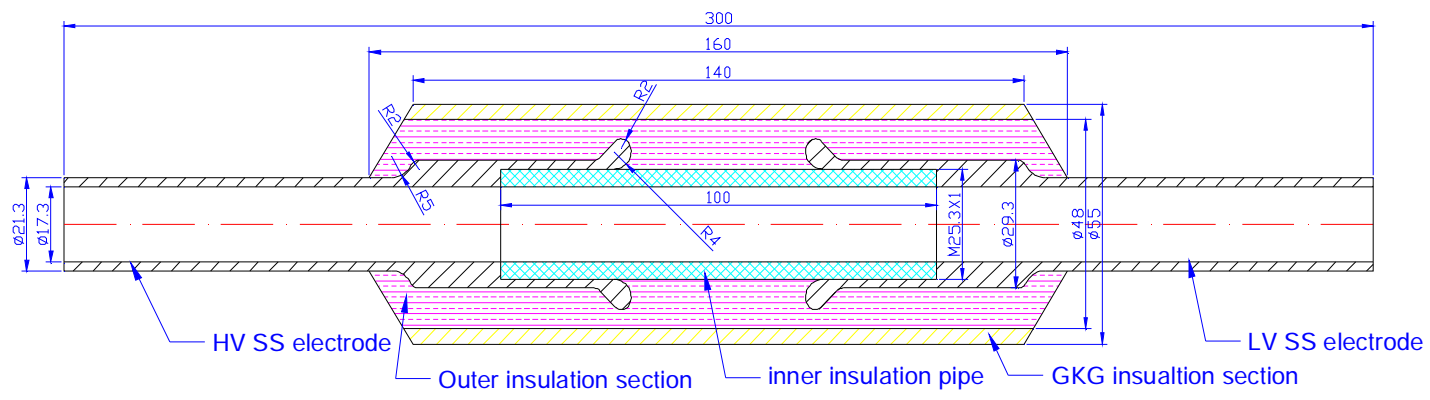

Fig. 1.The cross-section of the final design axial insulation break

The design of ITER axial insulation breaks are showed in Fig.1. The breaks consist essentially of an insulated tube between the two steel tubes inserted into the steel cryogenic line. The outer surface has a metal screen which is integrated into the ground screen of the high voltage cryogenic line, and there is metal reinforcing of both the insulating part, particularly where it overlaps the steel tubes to form a bond [3]. There length must be sufficient to prevent electrical breakdown between the two steel tubes through the fluid or gas. [4] 


\section{Threaded adhesive joints bonding GRP to Stainless Steel}

It is important that the contraction to low temperature on the outside diameter of the inner GRP tube should be less than the contraction of stainless steel to eliminate tensile strain in the bond. Bonding insulation tube to stainless tube using DWZ cryogenic resin was chosen as the method. The GRP contraction will be determined by the fiber content and winding angle and it is important these variables are controlled so that the bond is in compression at $4 \mathrm{~K}$.

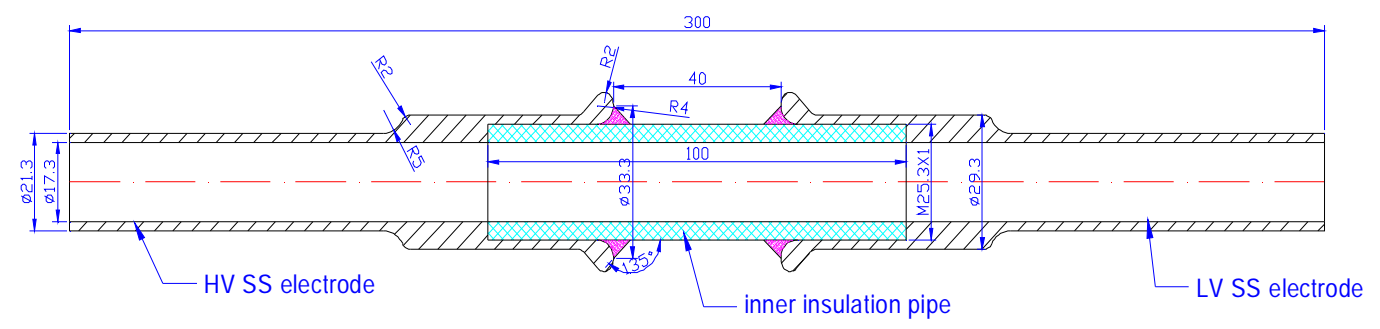

Fig.2readed adhesive joints bonding insulation tube to stainless tube

Threaded adhesive joints bonding insulation tube to stainless tube are showed in Fig.4. Process in detail is as follows: 1) Glue resin on bonding surface; 2) Rotate the inner tube into the electrodes by the thread; 3) Rotate back 2 circle and put glass tow into fitting area to keep no rich resin; 4) Rotate the inner tube into the electrodes and bond; 5) Cure under DWZ resin cure schedule. After preliminary test, the outer strengthening insulation structure will be fabricated by using the wet winding method.

\section{Interface electric field analysis}

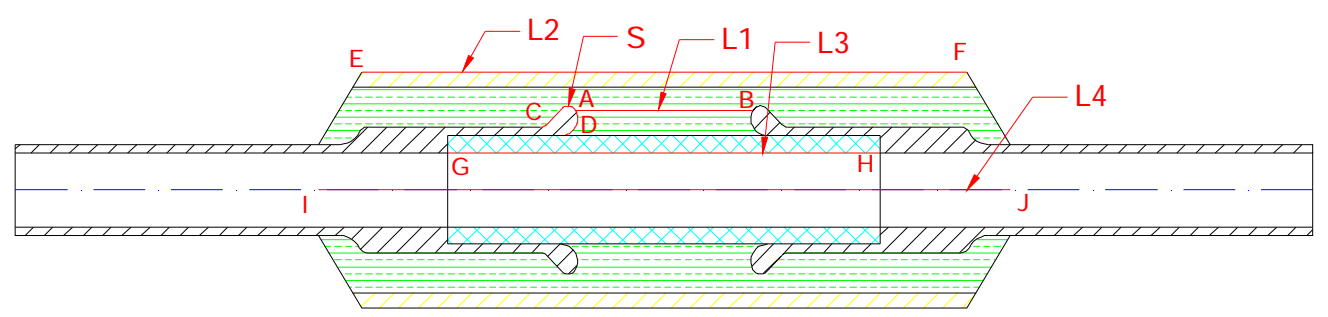

Fig.3.The sketch of interface profile distribution

The different electric field strength distribution paths are shown in Fig.3. The electric field strength distribution along S, L1, L2, and L3 path are shown in from Fig.4 to Fig.7. It is evident that the maximal value of electric field strength for insulation break is $3.396 \mathrm{kV} / \mathrm{mm}$ in Fig.4 and Fig.5, the maximal value of electric field strength along the outer interface between air medium and GFRP section is $1.231 \mathrm{kV} / \mathrm{mm}$ from Fig.6, and the maximal value of electric field strength along the surface of inner insulation pipe is $1.271 \mathrm{kV} / \mathrm{mm}$ from Fig.7, but the maximal value of electric field strength along the axial line of inner insulation pipe in air is only $1.203 \mathrm{kV} / \mathrm{mm}[5]$. 


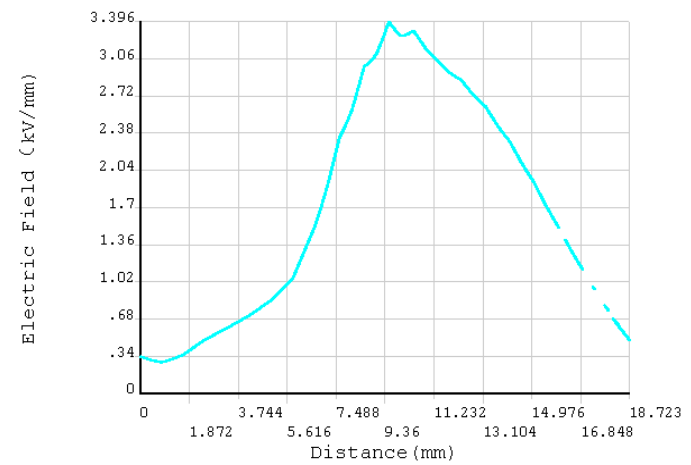

Fig.4.Electric field along $\mathrm{S}$ arc line from C, A to D

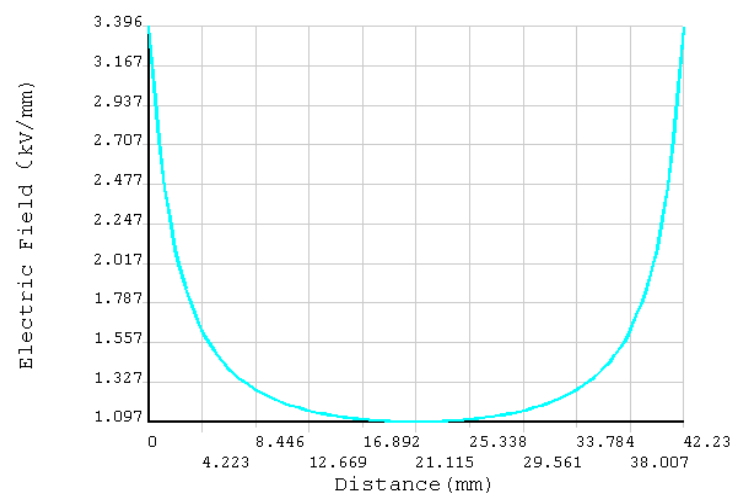

Fig.5.Electric field along L1 line from A to B

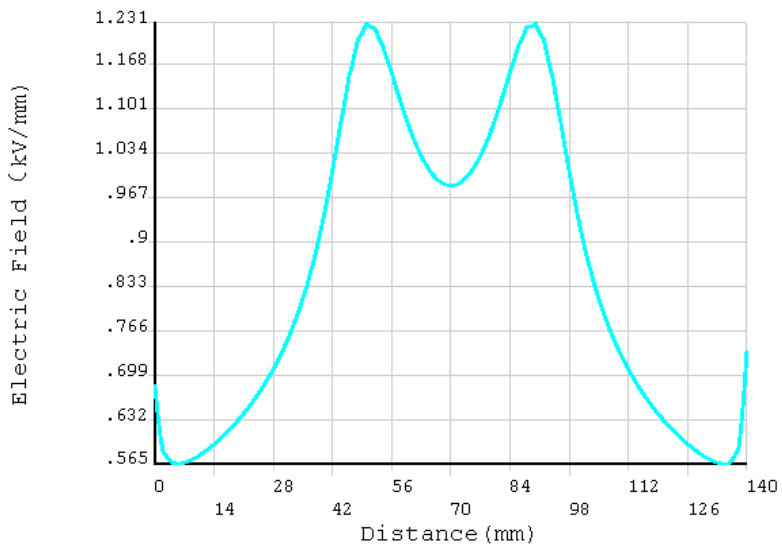

Fig.6.Electric field along L2 line from $\mathrm{E}$ to $\mathrm{F}$

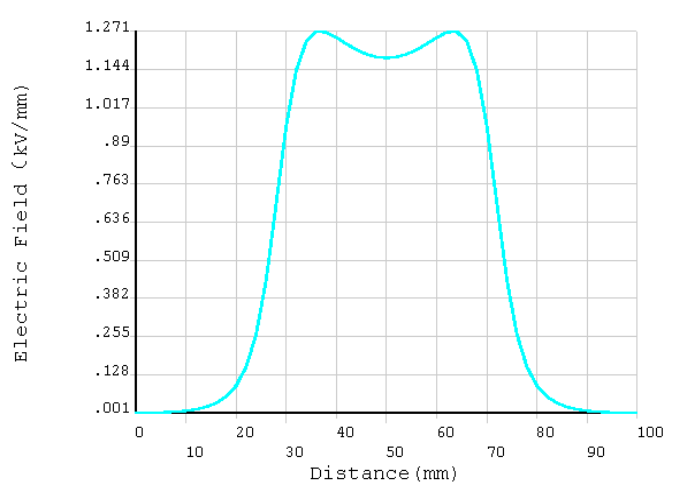

Fig.7 Electric field along L3 line from $\mathrm{G}$ to $\mathrm{H}$

\section{Conclusion}

Adhesive joints of axial insulation break was studied in detail. Wet winding process was chosen for 
Glass Fiber Reinforced Plastic, inner insulation tube, and threaded adhesive method was chosen for joints bonding GRP to Stainless Steel. It is fairly good results to eliminate the interface crack in low temperature heat shrinkage and mechanical stress to meet requirements for superconducting magnetic systems.

The electrical design analysis was conducted using the finite element method. The results showed the field strength of the axial break along the interface inner pipe is $1.23 \mathrm{kV} / \mathrm{mm}$, and the maximal field strength of the IBs locates on the top end of SS316L electrodes, the value of the maximum field strength is equal to $2.8 \mathrm{kV} / \mathrm{mm}$.

\section{Acknowledgements}

The research work was supported by National Natural Science Foundation of China under Grant No. 51667009 .

\section{References}

[1] Zhang Yuanbin, Pan Wanjiang. Theoretical and experimental analysis of cryogenic mechanics property for EAST Tokamak composite insulators [J]. Atomic Energy Science and Technology, 41(6), pp.752-755, 2007.

[2] Yang Changchun. Experiment research on low-temperature mechanic fatigue performance of ITER axial insulation breaks. Cryogenic \& Superconductivity, 39(11), pp.7-10, 2011.

[3] Yang Changchun Pan Wanjiang. Design and analysis on ITER axial insulation breaks. Nuclear Fusion and Plasma Physics, 30(3), pp.272-276, 2010.

[4] Wu Cheng, Pan Wanjiang, Yang Changchun. Research on performance test of ITER composite axial insulation breaks. Cryogenic \& Superconductivity, 39(10), pp.12-16. 2011.

[5] Yu X., Wu, W. Pan, W., Han S., Wang L., Wei, J. Liu L.; Du S., Zhou Z. Foussat, A.; Libeyre, P., Development of Insulation Technology with Vacuum-Pressure-Impregnation (VPI) for ITER Correction Coil. IEEE Transactions on Applied Superconductivity, 22 (3), pp. 212-206, 2012. 